\title{
Bose-Einstein condensation of metastable helium: some experimental aspects
}

\author{
C.I. Westbrook, A. Robert, O. Sirjean, A. Browaeys, D. Boiron, A. Aspect 円 $^{\text {A }}$ \\ Laboratoire Charles Fabry UMR 8501 du CNRS, B.P. 147, 91403 Orsay CEDEX, France
}

(Dated: October 28, 2018)

\begin{abstract}
We describe our recent realization of BEC using metastable helium. All detection is done with a microchannel plate which detects the metastables or ions coming from the trapped atom cloud. This discussion emphasizes some of the diagnostic experiments which were necessary to quantitatively analyze our results.
\end{abstract}

\section{INTRODUCTION}

Since the announcement of the observation of BoseEinstein condensation (BEC) in a rubidium vapor at the 12th ICOLS meeting in $1995 \mid 1]$, this fascinating state of matter has occupied central stage in the field of atomic, molecular and optical physics. The many new advances reported at this meeting indicate that the field may continue to do so for some time to come. This paper concerns one of these advances, the condensation of metastable helium atoms $\left(\mathrm{He}^{*}\right)$, and is intended as a supplement to the recently published Ref. [2]. We shall not repeat the data from that paper, but rather concentrate on some details that were left out of Ref. [2] for lack of space.

Until recently BEC had been observed in 4 different atomic species, $\mathrm{H}, \mathrm{Li}, \mathrm{Na}$ and $\mathrm{Rb}$, 3, 4, 5, 6], and the first question to ask before embarking on the quest for BEC of He* was whether a new atom was of interest. During the 1990s, several groups have been working on laser cooling of $\mathrm{He}^{*}$, and of course one answer to the above question is simply that the attainment of BEC is the best cooling one can do, and many of the same justifications for laser cooling apply to BEC. In addition, one might hope that a new atomic species might allow one to observe new phenomena, not accessible to the previously studied cases. In this respect it seemed clear that the metastability of the atoms might be very important. Simple, rapid and efficient detection of the $\mathrm{He}^{*}$ atoms is possible using electron multipliers such as microchannel plates (MCP) and these detectors can also be used to observe ions resulting from Penning ionizing collisions, either with residual gas atoms or between the $\mathrm{He}^{*}$ atoms themselves. Thus, when BEC was observed in alkali gases, groups working on laser cooling of $\mathrm{He}^{*}$ naturally considered the feasibility of $\mathrm{He}^{*}[7$.

A potential impediment to the achievement of the high densities necessary for evaporative cooling and BEC is the Penning ionization reaction:

$$
H e^{*}+H e^{*} \rightarrow H e+H e^{+}+e^{-} .
$$

Many experiments have shown that in a magneto-optical trap (MOT), this process is very rapid and limits the density in such a trap $8,9,10,11,12,13$. Thus, when

*http://atomoptic.iota.u-psud.fr/ loading atoms from a MOT into a non-dissipative trap, there was a danger that the density and more importantly the elastic collision rate would be too small to efficiently cool by evaporation. In addition, it was known experimentally [9, 10, 11] and theoretically [14] that even in the absence of resonant light, the rate constant for Penning ionization was on the order of $10^{-10} \mathrm{~cm}^{3} / \mathrm{s}$. Thus, even if it were possible to begin evaporative cooling, such a large inelastic collision rate is likely to prevent evaporative cooling down to BEC.

On the other hand it was predicted [18, 19] that the elastic scattering length $a$ would be quite large for He*. This result was very encouraging because it indicated that despite the low densities achievable in a MOT, thermalizing collisions in a MOT-loaded magnetic trap could be rapid enough to allow evaporative cooling.

Even more importantly, it was also known that, for a spin polarized sample, the Penning ionization rate is suppressed by spin conservation. He* has total angular momentum one, therefore only one trapping state exists and magnetically trapped $\mathrm{He}^{*}$ atoms are neccesarily $100 \%$ polarized. So one might hope to accumulate large densities of $\mathrm{He}^{*}$ in a magnetic trap. Experimentally, a suppression of one order of magnitude had already been demonstrated as early as 1972 15, this result was followed up by more recent measurements 16, 17. In the mid 1990's it was predicted that the degree of suppression could be as high as 5 orders of magnitude 18, 19, which would easily permit long storage times at densities necessary for BEC. The theoretical predictions for the scattering properties of $\mathrm{He}^{*}$ motivated the serious attempt to achieve BEC using a strategy analogous to that used in the alkali gases.

\section{EXPERIMENT}

In our apparatus, a Zeeman cooled atomic beam loads a MOT which, after an optical molasses cooling and an optical pumping stage, loads a magnetic trap. The magnetic trap is of the cloverleaf design 20. The only unusual feature of the apparatus is an MCP placed $5 \mathrm{~cm}$ below the trap center. A grid in front of the MCP allows one to either attract or repel positive ions. The front face of the MCP is at negative high voltage, and so electrons are never detected. We typically trapped $3 \times 10^{8}$ atoms in the MOT, and transferred approximately $50 \%$ to the 
magnetic trap. The MOT temperature was typically of order $1 \mathrm{mK}$, while after molasses cooling, the atomic temperature reached $300 \mu \mathrm{K}$. After loading the magnetic trap, the atomic sample was compressed by lowering the magnetic field bias. During this process the temperature increased again to about $1 \mathrm{mK}$. Then, an RF-knife was applied and ramped down from $130 \mathrm{MHz}$ to effect the forced evaporation.

The evolution of the temperature, phase space density and elastic collision rate during the evaporation ramp were all derived from measurements of the time of flight distribution of atoms to the MCP after the magnetic trap was turned off. Several examples are shown in Fig. 1. The change in gravitational energy of the atoms in falling $5 \mathrm{~cm}$ to the detector corresponds to $240 \mu \mathrm{K}$. For the initial temperature of the trapped atoms, this energy is negligible compared to the kinetic energy, and so the atoms expand nearly isotropically after release, and the collection efficiency of the detector corresponds roughly to its solid angle of $0.5 \%$. In this situation, the time of flight distribution is peaked at a value corresponding to the flight time of an atom moving toward the detector with the most probable speed at that temperature. The signature for cooling is a shift of the arrival time toward later times as can be seen in Fig. 1. Unfortunately, as the atoms are evaporatively cooled, their number also diminishes and the detected signal drastically decreases. Indeed when the temperature is of order $100 \mu \mathrm{K}$, no signal is visible on the detector. As the temperature decreases further however, the atoms begin to fall down rather than fly away, and the collection efficiency of the detector increases dramatically. Near a temperature of $10 \mu \mathrm{K}$ we observe a "revival" of the MCP signal, and at $1 \mu \mathrm{K}$, close to the BEC threshold, nearly all the atoms remaining in the trap reach the detector and we observe the characteristic structure of an expanding cloud below the BEC temperature: a broad peak whose width corresponds to the temperature and a narrow peak on top of it.

As is discussed in Ref. [2], a careful analysis of the narrow BEC peak reveals that its width increases as the $1 / 5$ power of the number of atoms $N_{0}$ in the peak as predicted by the Gross-Pitaevski equation in the ThomasFermi approximation. Upon closer examination however, the results were puzzling. First, it was surprising that we detected the atoms at $1 \mu \mathrm{K}$ at all. A magnetic field gradient as small as $30 \mathrm{mG} / \mathrm{cm}$ is enough to deviate the atomic trajectory so as to miss the MCP. We were virtually certain that residual field gradients larger than this were present in the apparatus. Secondly, the study of the expansion of the gas depends on being able to turn the trap off suddenly compared to the inverse of the oscillation frequencies in the trap $(50$ and $1300 \mathrm{~Hz})$. Here again we were certain from magnetic field measurements that effects such as eddy currents in the reentrant flanges holding the magnetic trap coils, limited our field turn-off time to about $1 \mathrm{~ms}$. Thirdly, we could estimate the number of atoms in the cloud at or near the critical temperature

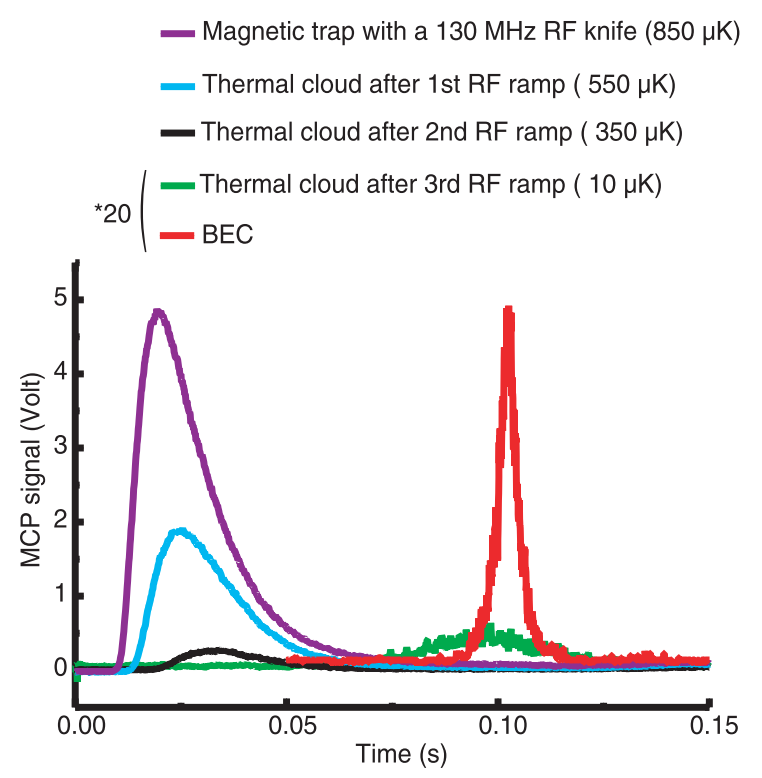

FIG. 1: MCP time of flight signals at different stages of the $\mathrm{RF}$ evaporation. The vertical scales on last two curves were multiplied by 20. At temperatures above $100 \mu \mathrm{K}$, the atomic cloud expands nearly isotropically, and the MCP detects primarily the downward going atoms. At temperatures low compared to $100 \mu \mathrm{K}$, the atoms fall in the earth's gravititional field and are nearly all detected. The mean arrival time is about $100 \mathrm{~ms}$, the time to fall $5 \mathrm{~cm}$. Because of the loss of atoms during evaporative cooling, and the small solid angle of the detector, very few atoms are detected at temperatures between 300 and $30 \mu \mathrm{K}$.

and compare it with the expected number in the ideal gas limit. The predicted number is given by [21]:

$$
N_{\mathrm{C}}=1.202\left(k T_{\mathrm{C}} / \hbar \tilde{\omega}\right)^{3} .
$$

Here $\tilde{\omega}$ denotes the geometric mean of the trap oscillation frequencies. Our calibration of the detector indicated a number of atoms smaller than this by an order of magnitude. Finally a quantitative analysis of the $N_{0}^{1 / 5}$ dependence, resulted in an estimate of the scattering length $a$ of order $100 \mathrm{~nm}$. Such a large value of the scattering length seemed to be ruled out by the fact that the condensate had a lifetime of a few $\mathrm{s}$. One expects the 3 body loss rate to scale as the fourth power of $a[22$, and therefore 3 body losses would have caused the BEC to decay in much less than $1 \mathrm{~s}$.

A clue to resolving these difficulties came from earlier, in situ measurements of the magnetic fields in our apparatus when the trap was turned off. In Fig. 2 we show the results of such a measurement. We began with atoms in a magnetic trap at $1 \mathrm{mK}$. While monitoring the MCP signal, we directed a $10 \mathrm{~mW} / \mathrm{cm}^{2}$ laser pulse of 20 $\mu$ s duration at the cloud at a time $t$ after the magnetic field turnoff. When the laser, which propagated parallel to the bias field, was resonant with the atoms, including the Zeeman shift due to the trapping fields, the atoms scattered the laser light and were pushed from the path 


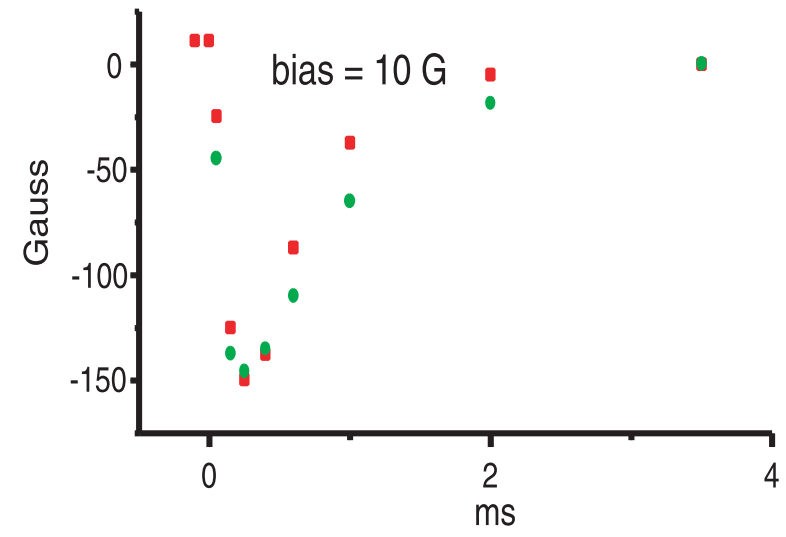

FIG. 2: In situ measurement of the magnetic field as a function of the time after turning off the magnetic trap. Black squares correspond to circular, grey circles to linear laser polarization. The field rapidly reverses due to differential eddy currents. This reversal causes approximately $10 \%$ of the atoms to transfer to the field-insensitive $m=0$ state.

to the detector. The laser detuning which minimized the MCP signal therefore corresponded to the Zeeman shift at the time of the pulse.

The magnetic field deduced from this Zeeman shift is plotted in Fig. 2 as a function of $t$. Negative times correspond to atoms still in the trap, and the value we observe agrees with the calculated magnetic field bias in the trap. One sees that the magnetic field in the trapping region undergoes a violent reversal during the turnoff, reaching a value of more than $-150 \mathrm{G}$ in less than $100 \mu \mathrm{s}$. This reversal is possibly due to the combined effects of the eddy currents induced by the pinch coils and the compensation coils. These two sets of coils carry currents in opposite directions and have very different sizes. The eddy currents they induce therefore have different signs and probably different time constants, which would explain the observed behavior. An uncompensated trap does not exhibit a magnetic field reversal.

It appears therefore that when the field reverses sign, some of the atoms undergo transitions to the field insensitive $m=0$ state. We presume that after the transition the atoms adiabatically remain in the $m=0$ state in the presence of the weak residual fields remaining in the apparatus. They therefore remain insensitive to any further field gradients.

We can test this interpretation by deliberately applying a magnetic field gradient with an external coil, and observing the time of flight spectrum. The results are shown in Fig. 3. The gradient was turned on about $100 \mu$ s after the currents to the magnetic trap were turned off. The figure shows two peaks, one arriving at approximately $100 \mathrm{~ms}$ after the field turnoff, as occurs without an applied gradient. The location and height of this peak does not depend on the magnitude of the gradient. The other peak occurs earlier, corresponding to atoms accelerated by the gradient and the arrival time shifts with the magnitude of the gradient. The height of the peak

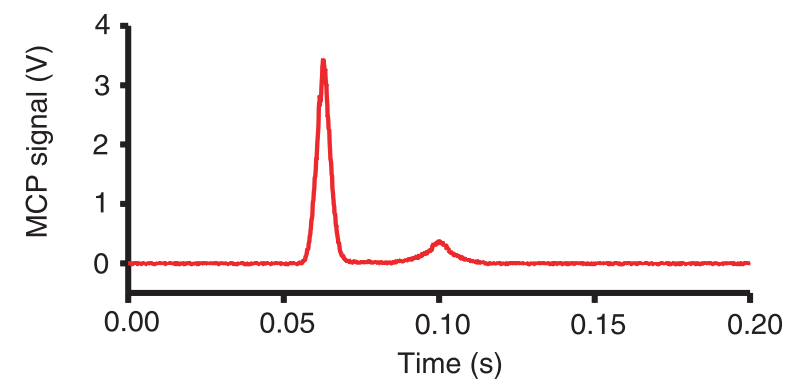

FIG. 3: Time of flight spectrum in the presence of a magnetic field gradient of an evaporatively cooled cloud of atoms. The height and arrival time of the small peak are independent of the applied gradient. The large peak's arrival time decreases as the vertical gradient (about $10 \mathrm{G} / \mathrm{cm}$ ) is increased. A 1 $\mathrm{G} / \mathrm{cm}$ horizontal gradient was also applied in order to maximize the number of atoms in the large peak. The ratio of the peak areas is 7 . Thus we believe that the small peak corresponds to atoms in the $m=0$ state, while the large peak corresponds to atoms in the $m=1$ state.

can be varied by varying the horizontal components of the gradient. The figure shows the largest early peak we were able to produce. These data show that indeed a fraction of the atoms makes the flight to the detector in the $m=0$ state and that at least 7 times more atoms are in a field sensitive state after the trap turnoff. The applied gradient was produced by a coil above the trap and thus the atoms which are accelerated are weak field seekers $(m=+1)$. It is also possible that some atoms are in the strong field seeking state $(m=-1)$, but since they are accelerated upwards, they have little chance to reach the MCP.

To get an estimate of the number of atoms in the magnetic trap we can use an analysis similar to that which leads to Eq. 1 leading to the number $N_{\text {th }}$ of atoms in the thermal cloud below the critical temperature. By fitting the wings of the time of flight spectra, we are able to determine the temperature $T$ of the atomic cloud, and to infer $N_{\text {th }}$. As discussed in Ref. 21], and experimentally demonstrated in Refs. 20, 23, 24], this number should be given by: $N_{\text {th }}=1.202(k T / \hbar \tilde{\omega})^{3}$. This relation gives an absolute thermodynamic measurement of the number of atoms. It is higher by a factor $f=8 \pm 4$ than the value we derive from the calibration of the MCP. Taking this correction into account, the largest condensate we have observed contained about $10^{5}$ atoms, and the number of atoms present at the critical temperature is a few times $10^{5}$.

The magnetic field measurements also help to explain why the analysis of the expansion of the trapped atoms after release works so well. Because of the fast reversal, the atoms which make transitions to the $m=0$ state are indeed released extremely rapidly. A careful analysis of the expansion may require taking into account the behavior of the weak field seeking atoms observed in Fig. 3. Here we assume that all atoms expand freely independent of their internal state. In fact the atoms 
in this state are presumably trapped during the decay of the eddy currents, but since in a cloverleaf trap, the confinement rapidly decreases with increasing bias, it is probably a good approximation to treat the atoms as free on the scale of $1 \mathrm{~ms}$.

An analysis of the mean field expansion of the cloud, using the corrected number of atoms leads to a value of the scattering length, $a=20 \pm 10 \mathrm{~nm}$. This result is consistent with our elastic rate constant measurements at $1 \mathrm{mK} 25]$, as well as with the observations of Ref. [26.

We have also observed the ions produced by the trapped condensate, by negatively biasing a grid above the MCP. An example of the ion detection rate as a function of time is shown in Fig. 4 of Ref. [2]. These ions are due to Penning ionization of residual gases, to two body collisions within the condensate, or possibly other, more complicated processes. We observe a factor of 5 more ions from the condensate than from a thermal cloud at 1 $\mu \mathrm{K}$, and we attribute this increase to the larger density in the condensate. The lifetime of the condensate, estimated by observing the ion rate is on the order of a few seconds. This is true both with and without an RF-knife to evacuate hot atoms 20, 27], although the lifetime is slightly longer with the knife present. The density of the condensate, deduced from its vertical size measurement and its known aspect ratio, is of order $10^{13} \mathrm{~cm}^{-3}$, so from the lifetime we can place an upper limit of $10^{-13} \mathrm{~cm}^{3} \mathrm{~s}^{-1}$ on the relaxation induced Penning ionization rate constant, as well as an upper limit of $10^{-26} \mathrm{~cm}^{-6} \mathrm{~s}^{-1}$ on any three-body loss process.

The achievement of BEC in $\mathrm{He}^{*}$ together with a $\mathrm{MCP}$ detector, offers many new possibilities for the investigation of BECs. Ion detection allows continuous "nondestructive" monitoring of the trapped condensate. We hope to be able to study the formation kinetics of the condensate using the ion signal. Our ability to count individual $\mathrm{He}^{*}$ atoms falling out of the trap should allow us to perform accurate comparisons of correlation functions 27 for a thermal beam of ultracold atoms 28 and for an atom laser, realizing the quantum atom optics counterpart of one of the fundamental experiments of quantum optics.

\section{Acknowledgments}

This work was supported by the European Union under grants IST-1999-11055, and HPRN-CT-2000-00125, and by the DGA grant 99.34 .050 .
[1] M. H. Anderson, J. R. Ensher, M. R. Matthews, C. E. Wieman, E. A. Cornell, Proceedings of the 12 International Conference on Laser Spectroscopy, Capri, June 1995, M. Ingucio, M. Allegrini and A. Sasso Eds., (World Scientific, Singapore, 1996).

[2] A. Robert, O. Sirjean, A. Browaeys, J. Poupard, S. Nowak, D. Boiron, C. I. Westbrook, A. Aspect, Science, 292, 463 (2001), published online 22 March 2001, 10.1126/science.1060622.

[3] M. H. Anderson, J. R. Ensher, M. R. Matthews, C. E. Wieman, E. A. Cornell, Science 269, 198 (1995).

[4] K. B. Davis et al., Phys. Rev. Lett. 75, 3969 (1995).

[5] C. C. Bradley, C. A. Sackett, J. J. Tollet, R. G. Hulet, Phys. Rev. Lett. 751687 (1995).

[6] D. G. Fried et al., Phys. Rev. Lett. 81, 3811 (1998).

[7] W. Vassen, OSA TOPS on Ultracold Atoms and BEC 7, 20, K. Burnett, Ed. (Optical Society of America, Washington, DC, 1996).

[8] F. Bardou, O. Emile, J. M. Courty, C. I. Westbrook, and A. Aspect, Europhys. Lett. 20, 681 (1992).

[9] H. C. Mastwijk, J. W. Thomsen, P. van der Straten, and A. Niehaus, Phys. Rev. Lett. 80, 5516 (1998).

[10] P. J. J. Tol, N. Herschbach, E. A. Hessels, W. Hogervorst, and W. Vassen, Phys. Rev A. 60, 761 (1999).

[11] M. Kumakura and N. Morita, Phys. Rev. Lett. 82, 2848 (1999).

[12] A. Browaeys, J. Poupard, A. Robert, S. Nowak, W. Rooijakkers, E. Arimondo, L. Marcassa, D. Boiron, C. I. Westbrook, and A. Aspect, Eur. Phys. J. D 8, 199 (2000).

[13] F. Pereira Dos Santos, F. Perales, J. Léonard, A. Sinatra, Junmin Wang, F. S. Pavone, E. Rasel, C. S. Unnikrish- nan, and M. Leduc, Eur. Phys. J. D 14, 15 (2001).

[14] V. Venturi and I. Whittingham, Phys. Rev. A, 61, 060703 (2000).

[15] J. C. Hill, L. L. Hatfield, N. D. Stockwell, and G. K. Walters, Phys. Rev. A 5, 189 (1972).

[16] N. Herschbach, P. J. J. Tol, W. Hogervorst, and W. Vassen, Phys. Rev. A 61, 050702(R) (2000).

[17] S. Nowak, A. Browaeys, J. Poupard, A. Robert, D. Boiron, C. I. Westbrook, and A. Aspect, Appl. Phys. B 70, 455 (2000).

[18] P. O. Fedichev, M. W. Reynolds, U. M. Rahmanov, G. V. Shlyapnikov, Phys. Rev. A 53, 1447 (1996); G. V. Shlyapnikov, T. M. Walraven, U. M. Rahmanov, M. W. Reynolds, Phys. Rev. Lett 73, 3247 (1994).

[19] V. Venturi, I. B. Whittingham, P. J. Leo, G. Peach, Phys. Rev. A 60, 4635 (1999), P. Leo, V. Venturi, I. Whittingham, J. Babb, preprint arXiv:physics/0011072.

[20] M. O. Mewes, M. R. Andrews, N. J. van Druten, D. M. Kurn, D. S. Durfee, and W. Ketterle, Phys. Rev. Lett. 77, 416 (1996).

[21] F. Dafolvo, S. Giorgini, L. P. Pitaevskii, Rev. Mod. Phys. 71, 463 (1999), and references therein.

[22] P. Fedichev, M. Reynolds, G. Shlyapnikov, Phys. Rev. Lett., 77, 2921 (1996).

[23] L. V. Hau et al., Phys. Rev. A 58, R54 (1998).

[24] J. R. Ensher, D. S. Jin, M. R. Matthews, C. E. Wieman, E. A. Cornell, Phys. Rev. Lett. 77, 4984 (1996).

[25] A. Browaeys, A. Robert, O. Sirjean, J. Poupard S. Nowak, D. Boiron, C.I. Westbrook, A. Aspect, Phys. Rev. A 64, 034703 (2001).

[26] F. Pereira dos Santos, J. Leonard, J. Wang, C. Barrelet, F. Perales, E. Rasel, C.S. Unnikrishnan, M. Leduc, C. 
Cohen-Tannoudji, Phys. Rev. Lett. 86, 3459 (2001).

[27] E. A. Burt et al., Phys. Rev. Lett. 79, 337 (1997).
[28] Y. Yasuda, F. Shimizu, Phys. Rev. Lett. 77, 3090 (1996). 